Erschienen in: Richard Schantz (Hg.), Die Philosophie der Wahrnehmung, in: Philosophical Analysis, ontos 2009, S. 211-228.

\title{
Die Wahrnehmung kausaler Prozesse
}

\author{
Thomas Grundmann, Köln
}

Abstract: In diesem Aufsatz wird die These (WKP) verteidigt, dass wir kausale Prozesse wahrnehmen können, und zwar in dem philosophisch interessanten Sinne, dass kausale Relationen in der Wahrnehmung phänomenal gegeben sind. Im ersten Teil werden zunächst drei Standardargumente Humes gegen (WKP) kritisiert. Im zweiten Teil werden die empirischen Untersuchungen des belgischen Psychologen Albert Michote herangezogen, um für (WKP) zu argumentieren. Besonders wichtig ist dabei die Einsicht, dass sich perzeptuelle Kausaleindrücke auch dann einstellen, wenn die Probanden wissen, dass keine kausale Relation vorliegt. Das spricht gegen eine epistemische Interpretation der Eindrücke als Wahrnehmungsmeinungen. Im dritten Teil wird schließlich ein anscheinendes K.O.-Argument gegen (WKP) diskutiert. Dieses Argument besagt, dass man kausale Relationen nicht wahrnehmen kann, weil man Tatsachen, die begrifflich durch die Kausalrelation impliziert werden (wie Naturgesetze oder kontrafaktische Abhängigkeiten), nicht wahrnehmen kann. Dieses Argument wird zurückgewiesen, indem gezeigt wird, dass Wahrnehmung nicht unter begrifflicher Implikation geschlossen ist. Abschließend wird der Versuch unternommen, positiv zu charakterisieren, was man wahrnimmt, wenn man kausale Relationen wahrnimmt. Es handelt sich um die Manifestation von dispositionalen Eigenschaften der beteiligten Gegenstände.

Wenn wir von den alltäglichen Beschreibungen unserer Wahrnehmung ausgehen, erscheint es nahezu selbstverständlich, dass wir kausale Prozesse wahrnehmen können. Wir sehen, wie eine Billardkugel eine andere anstößt. Wir sehen, dass ein schwerer Gegenstand auf ein weiches Kissen fällt und es eindellt. Wir sehen, wie ein Stein in ein Fenster fliegt und die Scheibe zertrümmert. Wir sehen, wie das Messer die Butter zerschneidet. Wir beobachten einen Unfall, bei dem ein Auto ein anderes eindrückt. Wir spüren, wie der Stoß durch eine andere Person uns aus dem Gleichgewicht bringt. In all diesen Fällen und vielen weiteren sehen oder beobachten wir, wie Ereignisse aufeinander einwirken. Dabei können wir auch selbst von einer solchen Einwirkung betroffen sein. Wir haben einen unmittelbaren Eindruck davon, dass ein Ereignis aktiven Einfluss auf ein anderes hat. Wir beobachten nicht nur, dass ein Ereignis auf ein anderes in der Zeit folgt. Wenn man sich klarmacht, wie viele der 
Prädikate, mit denen wir unsere Erfahrungen beschreiben, kausale Abläufe versteckt implizieren, dann sieht man, dass aus unserer Alltagsperspektive die Wahrnehmung kausaler Prozesse ein ubiquitäres Phänomen ist. Sobald wir sagen, dass wir sehen, wie ein Gegenstand einen anderen trägt, stößt, drückt, verdrängt oder dazu beiträgt, dass er sich irgendwie verändert, gehen wir davon aus, dass wir kausale Prozesse wahrnehmen. ${ }^{1}$

Es ist deshalb zunächst einmal erstaunlich, dass es unter Philosophen seit Hume als ausgemacht gilt, dass wir kausale Prozesse nicht wahrnehmen können. In seiner Untersuchung über den menschlichen Verstand resümiert Hume:

„Es zeigt sich, dass wir in Einzelfällen der Wirksamkeit von Körpern (...) nie etwas anderes entdecken konnten, als dass ein Ereignis dem anderen folgt; aber wir sind nicht imstande, irgendwelche Kraft oder Macht zu begreifen, durch welche die Ursache wirkt, oder irgend eine Verknüpfung zwischen ihr und der angenommenen Wirkung. (...) so das, im Ganzen genommen, überall in der ganzen Natur sich nicht ein einziges Beispiel von Verknüpfung darbietet, das uns vorstellbar wäre. Alle Ereignisse erscheinen durchaus unzusammenhängend und vereinzelt. Ein Ereignis folgt dem anderen; aber nie können wir irgendein Band zwischen ihnen beobachten. Sie scheinen zusammenhängend, doch nie verknüpft (...).“ (Hume 1993, S. 89f) Um diese Diskrepanz zwischen unserem alltäglichen Selbstverständnis und der herrschenden philosophischen Meinung besser verstehen zu können, soll in einem ersten Schritt geklärt werden, was kausale Prozesse sind und unter welchen Bedingungen es wahr wäre, dass wir sie wahrnehmen können.

Ich möchte zunächst die Frage nach den kausalen Prozessen aufgreifen. Ein kausaler Prozess besteht in einer Beziehung zwischen zwei oder mehreren Einzelereignissen. Der Stoß einer ganz bestimmten Billardkugel ist kausal verantwortlich dafür, dass sich eine zweite Billardkugel mit einer bestimmten Geschwindigkeit und in einer ganz bestimmten Richtung nach dem Anstoß bewegt. Von so genannten Singularisten ist die These vertreten worden, dass es für eine kausale Relation zwischen zwei Ereignissen hinreicht, wenn sie zeitlich unmittelbar aufeinander folgen und räumlich direkt aneinander grenzen (hier spricht man auch von Kontiguität). ${ }^{2}$ Doch bereits Hume hatte darauf hingewiesen, dass eine Kausalrelation neben zeitlicher Folge und Kontiguität zusätzlich eine „,notwendige Verbindung“ erfordert. Zeitliche und räumliche Kontiguität können für Kausalität nicht ausreichen, da sie auch von

\footnotetext{
${ }^{1}$ Vgl. dazu Anscombe 1971.

${ }^{2}$ Vgl. Ducasse 1926 und Anscombe 1971.
} 
vielen Epiphänomenen erfüllt werden. Bei einer Erkältung geht das Frösteln dem Fieber unmittelbar voraus und betrifft auch denselben Körper. Dennoch sind beide Phänomene gemeinsame Wirkungen einer Infektion. Außerdem können wir uns eine Welt zumindest vorstellen, in der nur zwei Ereignisse existieren, die unmittelbar aufeinander folgen und direkt räumlich aneinander grenzen und dennoch kausal nicht miteinander verknüpft sind, weil Gott sie unmittelbar nebeneinander und nacheinander erschafft. Wenn dies aber vorstellbar ist, dann kann Kontiguität Kausalität nicht begrifflich implizieren. Es ist deshalb sehr plausibel, dass über ihre zeitliche und räumliche Kontiguität hinaus mehr dazu erforderlich ist, damit zwei Ereignisse kausal verknüpft sind. Die kausale Abhängigkeit der Wirkung von der Ursache wird von vielen Theorien entweder durch das Bestehen von Gesetzen erklärt, unter die die Ereignisse fallen, oder durch kontrafaktische Beziehungen der Art, dass die Wirkung nicht aufgetreten wäre, wenn sich die Ursache nicht ereignet hätte. Es kann an dieser Stelle offen bleiben, wie die Zusatzbedingung genau lautet, wichtig ist nur, dass es eine solche Zusatzbedingung geben muss. Kontiguität alleine reicht nicht aus.

Was genau wird nun behauptet, wenn man sagt, dass kausale Prozesse wahrnehmbar sind? Würde damit nur behauptet, dass kausale Prozesse de re wahrnehmbar sind, dann wäre die These trivial. Wir können selbstverständlich Wahrnehmungen von Ereignisfolgen haben, die de facto kausal miteinander verknüpft sind. In diesem Sinne kann man auch dann einen bestimmten Zwilling de re wahrnehmen, wenn für einen beide Zwillinge völlig gleich aussehen. Man nimmt den einen Zwilling de re wahr, wenn die Wahrnehmung, mit welchem Gehalt auch immer, faktisch von diesem Zwilling hervorgerufen wurde. Wenn behauptet wird, dass kausale Prozesse wahrnehmbar sind, dann will man damit jedoch sagen, dass kausale Prozesse in den de dicto Gehalt der Wahrnehmung gehören. Die kausale Relation zwischen den Ereignissen soll dem Betrachter also selbst phänomenal gegeben sein. Er soll nicht nur von einem kausalen Prozess einen Eindruck haben, sondern wahrnehmen, dass dieser kausale Prozess besteht.

Ein zweiter Punkt: Häufig sagen wir, dass wir etwas wahrnehmen, wenn wir aufgrund von beobachtbaren Indizien unmittelbar zu einem Urteil oder einer Meinung über die Sache kommen. In diesem Sinne sagen wir auch, dass der Wissenschaftler durch seinen Blick auf die Messgeräte mikrophysikalische Prozesse beobachtet. Tatsächlich sind die mikrophysikalischen Prozesse aber nicht der unmittelbare Inhalt seiner Wahrnehmung (dieser bezieht sich auf die Anzeige des von ihm beobachteten Geräts), sondern der Inhalt einer 
Meinung, die er aufgrund seiner Beobachtung erwirbt. Wenn wir Beobachtungsmeinungen im Blick haben, dann verwenden wir das Wahrnehmungsprädikat im epistemischen Sinne. In der Diskussion der Frage, ob kausale Prozesse wahrnehmbar sind, geht es jedoch darum zu klären, ob kausale Prozesse zum phänomenalen Inhalt der Wahrnehmung selbst werden können. Ist Kausalität phänomenal erfahrbar und erlebbar? Um diese Frage zu klären, müssen wir von den Beobachtungsmeinungen ganz abstrahieren.

Schließlich werden Wahrnehmungszuschreibungen normalerweise in einem faktiven Sinne verwendet. Das heißt: Wenn jemand etwas wahrnimmt, dann existiert es auch. Nichtexistierende Dinge oder Ereignisse können nicht wahrgenommen werden. Darum soll es hier nicht gehen. Wenn ich der Frage nachgehe, ob kausale Prozesse wahrnehmbar sind, dann möchte ich mich damit nicht darauf festlegen, dass es kausale Prozesse in der Welt tatsächlich gibt. (Das ist natürlich sehr wahrscheinlich.) Sondern es geht allein um die Frage, ob kausale Prozesse zum repräsentationalen oder intentionalen Inhalt von phänomenalen Erlebnissen gehören. Dieser Inhalt lässt es zunächst einmal offen, ob er erfüllt oder nicht erfüllt ist.

Auf dem Hintergrund der bisherigen Vorüberlegungen lässt sich die These der Wahrnehmbarkeit kausaler Prozesse jetzt etwas präziser formulieren:

(WKP)Es gibt Sinneswahrnehmungen, die in ihrem repräsentationalen Inhalt unmittelbar von kausalen Prozessen handeln. Sie repräsentieren die beteiligten Ereignisse als raumzeitlich aneinandergrenzend und als voneinander abhängig.

Es ist klar, dass wir über die Wahrheit oder Falschheit dieser These nicht so ohne weiteres aufgrund unseres Alltagsverständnisses urteilen können. So könnte es leicht sein, dass wir von der Wahrnehmbarkeit kausaler Prozesse bereits dann sprechen, wenn wir Beobachtungsmeinungen über kausale Prozesse haben. Die Wahrnehmbarkeit wäre dann nur epistemisch. Und dies ist mit der Falschheit der These verträglich.

Im Folgenden möchte ich die These (WKP) verteidigen. Selbstverständlich möchte ich nicht bestreiten, dass es viele kausale Prozesse gibt, die für uns nicht wahrnehmbar sind. Viele Mikroprozesse sind einfach zu klein, um von uns direkt beobachtet werden zu können. Das wird man schwerlich leugnen können. Ich will nur behaupten, dass es kausale Prozesse gibt, die wir beobachten können und auch tatsächlich beobachten. So ließe sich erklären, wie wir den Kausalbegriff empirisch erwerben können, um ihn dann auch in unseren kausalen Schlüssen, Hypothesen und funktionalen Charakterisierungen verwenden zu können. Ich 
werde zunächst in einem ersten Abschnitt Humes Einwände gegen die Wahrnehmbarkeit kausaler Prozesse darstellen und zurückweisen. In einem zweiten Abschnitt werde ich dann positiv dafür argumentieren, dass der repräsentationale Gehalt unserer phänomenalen Erlebnisse von kausalen Prozessen handelt. Schließlich werde ich mich im letzten Abschnitt dem wohl härtesten Einwand gegen meine These zuwenden. Dieser Einwand besagt, dass kausale Prozesse nicht wahrnehmbar sind, weil konstitutive Bestandteile der Kausalrelation nicht wahrnehmbar sind. Ich werde zu zeigen versuchen, dass auch dieser Einwand zurückgewiesen werden kann.

David Hume hat vehement bestritten, dass wir mehr als eine zeitliche Aufeinanderfolge und ein räumliches Aneinandergrenzen zweier Ereignisse wahrnehmen können. Eine dynamische (notwendige) Verbindung zwischen den Ereignissen werde prinzipiell nicht wahrgenommen. Deshalb kann man nach Hume kausale Prozesse nicht wahrnehmen. Der Eindruck von Kausalität entstehe vielmehr dadurch, dass der Betrachter aufgrund von Gewohnheit beim Erlebnis eines Ereignisses das Eintreten eines zweiten Ereignisses erwartet, das regelmäßig zusammen mit dem ersten Ereignis aufgetreten ist. Es handelt sich also nur um eine subjektive Projektion, dem nichts in den beobachteten Eigenschaften selbst zugrunde liegt.

Es lassen sich insgesamt drei Argumente aus den Texten Humes rekonstruieren, die gegen die Wahrnehmbarkeit eines kausalen Nexus zwischen zwei Ereignissen sprechen. Sein erstes Argument ist eine reductio ad absurdum der Annahme einer notwendigen Verbindung zwischen Ursache und Wirkung. Der Grundgedanke des Arguments ist folgender: Damit ein notwendiger Zusammenhang zwischen Ursache und Wirkung erfahren werden kann (dass also die Ursache die Wirkung beispielsweise erzwingt), muss die Wirkung nach Hume aus der Ursache ohne weitere Erfahrung a priori ableitbar sein. Das ist aber tatsächlich nie der Fall. Also kann kein notwendiger Zusammenhang zwischen Ursache und Wirkung erfahren werden. Im Originaltext heißt es:

„Aus der ersten Erscheinung eines Gegenstandes lässt sich nie mutmaßen, welche Wirkung aus ihm entspringen wird. (...) Unmöglich kann daher die Vorstellung der Kraft von der Betrachtung der Körper in Einzelfällen ihrer Tätigkeiten herstammen (...).“ (Hume 1993, S. 77f)

Etwas formaler lautet Humes Argument so: 
(1) Wenn zwei Ereignisse als notwendig zusammenhängend erfahren werden, dann muss die Wirkung a priori aus der Erfahrung der Ursache ableitbar sein.

(2) Die Wirkung ist nicht a priori ableitbar aus der Erfahrung der Ursache.

Also: Ereignisse können nicht als notwendig zusammenhängend erfahren werden.

Die Prämisse (2) ist offenkundig richtig. Solange wir keine Erfahrung über die Wirkungen eines Dinges gemacht haben, können wir nicht sagen, welche Wirkungen es haben wird. Die dispositionalen Kräfte eines Dinges kennen wir empirisch nur aus seiner Interaktion mit anderen Dingen. Aber Prämisse (1) sieht dubios aus. Warum sollte es nicht metaphysisch notwendige Zusammenhänge zwischen Ereignissen geben, die wir eben nur aus der Erfahrung der Beziehung zwischen den Ereignissen kennen? Auch andere metaphysisch notwendige Tatsachen, etwa dass Wasser notwendig $\mathrm{H} 2 \mathrm{O}$ ist, kennen wir nur a posteriori aus der Erfahrung. Nicht jeder notwendige Zusammenhang ist rein begrifflicher Natur. ${ }^{3}$

Hume nennt noch ein weiteres Argument gegen die Wahrnehmbarkeit kausaler Prozesse: das Argument von der Geschlossenheit der Wahrnehmung unter ontologischer Realisierung. Auch dieses Argument hat die Form einer reductio. Hier zunächst wieder die Grundidee: Wenn wir kausale Prozesse wahrnehmen könnten, dann müssten wir auch ihre ontologische Realisierung wahrnehmen und kennen. Aber wir kennen ihre ontologische Realisierung nicht. Deshalb können wir kausale Prozesse auch nicht wahrnehmen. Hume verwendet dieses Argument gegen die Wahrnehmbarkeit innerpsychischer Kausalprozesse (wenn der Wille unsere Vorstellungen kontrolliert). Im Originaltext heißt es:

„Wir empfinden nur das Ereignis, nämlich das Vorhandensein einer Vorstellung als Folge eines Willensbefehls; aber die Art, in der dieser Vorgang sich vollzieht, die Kraft, durch die er hervorgebracht wird, übersteigt völlig unser Verständnis.“ (Hume 1993, S. 83)

In meiner Rekonstruktion lautet Humes Argument dann folgendermaßen:

(1) Wenn wir etwas wahrnehmen, dann nehmen wir auch die Tatsachen wahr, die dieses Ding ontologisch realisieren.

(2) Wir nehmen die Realisierer kausaler Prozesse niemals wahr.

Also: Wir nehmen kausale Prozesse niemals wahr.

\footnotetext{
${ }^{3}$ Vgl. zu dieser Kritik auch Menzies 1998.
} 
Auch in diesem Argument ist Prämisse (2) sehr einleuchtend. Selbst jemand, der die Wahrnehmung makroskopischer kausaler Prozesse propagiert, wie etwa den Umschlag der Farbe im Reagenzglas durch Beimischung bestimmter Substanzen, würde kaum behaupten, dass wir auch die mikrophysikalischen Kausalprozesse alle erfassen, die diesen makroskopischen Vorgang konstituieren. Aber Prämisse (1) ist nicht plausibel. Ein ganz einfaches Beispiel kann das zeigen: Ich nehme den Tisch vor mir wahr. Der Tisch ist durch ein Aggregat vieler mikrophysikalischer Tatsachen konstituiert. Gleichwohl nehme ich diese konstituierenden oder realisierenden Tatsachen nicht wahr. Die Wahrnehmung ist also unter ontologischer Realisierung nicht geschlossen, wie Prämisse (1) fälschlich unterstellt.

Sehen wir uns schließlich noch ein drittes Argument an, das ich so nicht direkt bei Hume gefunden habe, das aber analog zu seinem Illusionsargument für Sinnesdaten als unmittelbarem Gegenstand der Wahrnehmung formuliert werden könnte. Ich möchte es als Illusionsargument gegen die Wahrnehmbarkeit kausaler Prozesse bezeichnen:

(1) Ich habe den Eindruck von Kausalität auch im Fall einer bloßen Kontiguität von Ereignissen ohne echte kausale Verknüpfung.

(2) In solchen illusorischen Fällen kann sich der Eindruck nicht auf eine kausale Relation beziehen (denn die existiert nicht), sondern nur auf reine Abfolgen von räumlich aneinandergrenzenden Ereignissen.

(3) Veridische Kausaleindrücke lassen sich nicht von illusorischen Eindrücken unterscheiden und deshalb beziehen auch sie sich nicht auf kausale Prozesse.

Also: Der Sinneseindruck bezieht sich niemals auf kausale Prozesse (sondern gibt nur Anlass zu wahren oder falschen Kausalurteilen).

Genau wie in allen anderen Illusionsargumenten ist in diesem Argument die Annahme (Prämisse 2) falsch, also die Annahme, dass sich ein Eindruck nicht auf ein nichtexistierendes Objekt beziehen kann. Aufgrund von intentionalem Inhalt ist eine solche Beziehung auf Nicht-Existierendes möglich. Und deshalb kann man gegen den humeschen Geist dieses Arguments daran festhalten, dass selbst illusorische Kausaleindrücke von kausalen Prozessen handeln, obwohl es sich um Fehlrepräsentationen handelt. 
Dieser kurze, zugegeben kursorische Durchgang durch Humes Argumente gegen die Wahrnehmbarkeit kausaler Prozesse zeigt, dass diese Argumente bei weitem nicht so zwingend sind, wie die Tradition angenommen hat, wenn es nach Hume quasi als Sakrileg galt, kausale Prozesse für wahrnehmbar zu halten. Im folgenden Abschnitt möchte ich mir nun die positiven Argumente für die Wahrnehmbarkeit kausaler Prozesse ansehen.

Bereits in den 40er Jahren hat der belgische Psychologe Albert Michote eine Reihe von höchst aufschlussreichen Experimenten zur kausalen Wahrnehmung durchgeführt. In weit über 100 unterschiedlichen Experimenten wurden Probanden bewegte Objekte präsentiert und sie sollten berichten, ob sie einen kausalen Einfluss der Objekte aufeinander wahrnehmen. Besonders aufschlussreich waren die so genannten „Launching“-Experimente. Dabei bewegt sich ein Objekt auf anderes, unbewegtes Objekt zu und nach der Berührung bewegt sich das zweite Objekt in dieselbe Richtung wie das erste. Die Probanden berichteten in diesen Fällen, dass sie den Eindruck hätten, dass das erste Objekt das zweite anschiebt und in Bewegung setzt. Sie hatten den Eindruck von einer kausalen Interaktion zwischen den Objekten. Durch eine geschickte Variation der Stimuli fand Michote heraus, dass zur Auslösung des kausalen Eindrucks bestimmte Bedingungen erfüllt sein müssen. Die Objekte müssen sich berühren. Der Zeitraum zwischen der Berührung der Objekte und der Bewegung des zweiten Objekts muss relativ gering sein. Und die Bewegungsrichtung des zweiten Objekts muss der des ersten Objekts entsprechen. Diese Daten lassen sich so zusammenfassen, dass zu den notwendigen Auslösungsbedingungen eines kausalen Eindrucks die räumliche und zeitliche Kontiguität der Ereignisse gehört.

Außerordentlich interessant war die Entdeckung, dass sich der kausale Eindruck auch dann bei den Probanden einstellte, wenn sie im Voraus wussten, dass eine wirkliche kausale Interaktion nicht stattfand. Michote berichtete ,that the causal impression was not necessarily dependent on the use of ,real' solid objects. It can be produced perfectly clearly by using objects which are simple coloured shapes without apparent thickness, or even images projected on a screen, and this is possible even when the observers know perfectly well what is going on." (Michote 1963, S. 84) Hier haben wir einen ganz ähnlichen Experimentalbefund wie im Falle der Müller-Lyer-Illusion. Auch bei der Müller-Lyer-Illusion stellt sich der Eindruck ein, dass die Linien verschieden lang sind, selbst wenn wir wissen, dass sie gleich 
lang sind. Das spricht dafür, dass es sich bei diesem Eindruck nicht um ein Beobachtungsurteil handelt (denn das wäre bei gegenteiligem Wissen klar irrational), sondern um einen phänomenalen Eindruck, der robust gegenüber von Zusatzinformationen ist. Wenn sich der Eindruck eines kausalen Prozesses also auch dann einstellt, wenn die Probanden wissen, dass sie nur Projektionen oder flächige Farbensembles sehen, dann spricht das eindeutig dafür, dass der kausale Eindruck zum repräsentationalen Gehalts der Erfahrung selbst gehört und nicht auf einer Meinung beruht. Die epistemische Interpretation des Phänomens kann also ausgeschlossen werden.

Michote konnte außerdem experimentell Humes psychologische Erklärung des Kausaleindrucks widerlegen. Wie bereits früher hervorgehoben, war Hume der Auffassung, dass der Eindruck einer Verbindung zwischen Ursache und Wirkung nicht durch die Wahrnehmung selbst entsteht. Sie hat keine solche Verbindung zum Inhalt. Sondern für Hume entsteht dieser Eindruck einer Verbindung durch die Erwartung des nachfolgenden Eintretens eines Ereignisses von einem bestimmten Typ aufgrund einer in der Vergangenheit beobachteten Korrelation von Ereignissen. Deshalb können wir nach Hume niemals den Eindruck einer kausalen Interaktionen zwischen Ereignissen haben, die noch nie zusammen aufgetreten sind, sondern unerwartet aufeinander folgen:

„Hiernach scheint es, dass die Vorstellung einer notwendigen Verknüpfung von Ereignissen ihren Ursprung in einer Häufung eingetretener gleichartiger Fälle hat, in denen beständig diese Ereignisse im Zusammenhang standen; ein einzelner solcher Fall kann nie jene Vorstellung eingeben (...).“ (Hume 1993, S. 91)

Michote konnte nun in seinen Experimenten nachweisen, dass sich der Kausaleindruck auch in Fällen ergab, die völlig neuartig waren und keine in der Vergangenheit beobachtete Korrelation ähnlicher Ereignisse voraussetzen. (Michote 1963, S. 263) Es genügen jedoch auch Fälle aus der Alltagserfahrung, um $\mathrm{zu}$ zeigen, dass ein perzeptueller Wahrnehmungseindruck nicht die Beobachtung einer regelmäßigen Korrelation voraussetzt. Nehmen Sie an, Sie öffnen die Tür Ihres Kühlschranks und genau in diesem Moment geht das Licht in Ihrer Wohnung aus. Dann kann sich der Eindruck einstellen, als ob das Öffnen der Kühlschranktür das Ausgehen des Lichtes bewirkt. Dieser Effekt tritt auch dann ein, wenn Sie nie zuvor eine solche Abfolge von Ereignissen beobachtet haben. Also kann der kausale Eindruck nicht, wie Hume behauptet, das Resultat einer Projektion aus vergangenen Beobachtungen sein. 
Fassen wir zusammen: Michotes Experimente legen nahe, dass der kausale Eindruck bei der Beobachtung von Interaktionen zwischen Objekten zum Inhalt der Wahrnehmung selbst gehört und dass es sich bei diesem Inhalt nicht um den Inhalt einer Beobachtungsmeinung handelt, sondern um den repräsentationalen Phänomengehalt der Wahrnehmung selbst. Es bleibt die Frage, ob Michotes Nachweis, dass die räumliche und zeitliche Kontiguität von Ereignissen den Kausaleindruck auslöst, nicht zeigt, dass der vermeintliche Kausaleindruck tatsächlich nur von einer solchen Kontiguität der Ereignisse handelt. Das wäre jedoch klarer Weise ein Fehlschluss. Die Auslösebedingungen eines mentalen Zustands dürfen auf keinen Fall einfach mit dem Inhalt dieses Zustands identifiziert werden. Ansonsten wären Illusionen (und Fehlrepräsentationen) ganz unerklärlich. So kann z.B. durch die Müller-Lyer Pfeile, die tatsächlich gleichlang sind, der Eindruck hervorgerufen werden, dass die Pfeile unterschiedlich lang sind. Wären die Auslösebedingung des Eindrucks mit seinem Inhalt identisch, dann könnte der Eindruck der unterschiedlichen Länge so gar nicht erklärt werden. Denn die Pfeile sind ja gleich lang und rufen den Eindruck unterschiedlicher Länge hervor. Dass der Kausaleindruck mehr als bloß die Kontiguität der Ereignisse beinhaltet, lässt sich auch phänomenologisch plausibel machen. Stellen Sie sich vor, Sie sehen eine Billardkugel, die sich ohne Effet direkt auf eine zweite unbewegte Billardkugel zu bewegt, diese in der Mitte trifft und unmittelbar danach bewegt sich diese zweite Billardkugel mit etwa der gleichen Geschwindigkeit in dieselbe Richtung wie die erste. Wenn Sie das sehen, dann entsteht bei Ihnen sofort der Eindruck einer kausalen Einwirkung der ersten auf die zweite Kugel. Nehmen Sie jetzt an, dass die Bewegung der zweiten Kugel tatsächlich (aber für Sie unbemerkbar) gar nicht durch den Anstoß der ersten Kugel hervorgerufen wurde. Tatsächlich werden beide Kugeln durch einen verborgenen Mechanismus von starren Läufern bewegt. Wenn beide Kugeln sich berühren, stoppt dieser Mechanismus die Bewegung der ersten Kugel $\mathrm{ab}$ und setzt die zweite Kugel in Bewegung. Wenn Sie diese Zusatzinformation bekommen, dann werden Sie denken, dass ihr Wahrnehmungseindruck falsch war. Da in der konkreten Situation jedoch eine Kontiguität der beiden Ereignisse vorlag, ist das nur erklärbar, wenn der Inhalt Ihrer Wahrnehmung mehr umfasste als die bloße Kontiguität der Ereignisse.

Es bleibt dennoch die Frage offen, wie die Auslösebedingungen eines Sinneseindrucks mit seinem Inhalt zusammenhängen. Betrachten wir eine mögliche Option: Informationale Theorien des Inhalts besagen, dass der Inhalt eines mentalen Zustands durch seine Ursachen bestimmt wird. Um Fehlrepräsentationen $\mathrm{zu}$ ermöglichen, dürfen nicht die jeweiligen 
Kausalursachen der Zustände deren Inhalt bestimmen, sondern es müssen die historischen Ursachen von Zuständen desselben Typs wie der fragliche Zustand sein. Man kann den Gehalt beispielsweise durch die normale Ursache von Zuständen eines Typs bestimmt sehen. Oder durch die Ursache in der Situation, in der der Zustand seinen Inhalt erwarb. Es ist hier relativ egal, wie wir die Situation, in der die Ursache den Inhalt bestimmt, genauer charakterisieren. Wichtig ist nur, dass in diesen gehaltskonstitutiven Situationen Ereignisse, die in einer Kontiguitätsrelation zueinander stehen, auch kausal miteinander interagieren. Wenn das der Fall ist, dann können Zustände, die durch kontiguierliche Ereignisse ausgelöst werden, die kausale Verbindung dieser Ereignisse zu ihrem Inhalt haben. Dass räumlich und zeitlich unmittelbar aneinandergrenzende Ereignisse unter Normalbedingungen auch in einer kausalen Relation zueinander stehen, ist nun aber ziemlich plausibel. So kann durch die Korrelation von Kontiguität und Kausalität in den relevanten Situationen erklärt werden, warum Sinneseindrücke, die durch kontiguierliche Ereignisse ausgelöst werden, kausale Prozesse zum Inhalt haben.

III

Solange man annimmt, das die kausale Relation nur in einer singulären Relation zwischen Ursache und Wirkung besteht und von keinerlei weiteren Tatsachen abhängt, scheint der These, dass man kausale Prozesse wahrnehmen kann, nichts mehr weiter im Wege zu stehen, zumindest wenn man dabei nur makroskopische Prozesse im Auge hat. Der so genannte Singularismus (Ducasse, Anscombe, Menzies) ist also sehr gut verträglich mit der Wahrnehmbarkeit kausaler Prozesse. Doch viele der herkömmlichen Theorien der Kausalität beinhalten weitere problematische Voraussetzungen der Kausalbeziehung. So vertreten Regularitätstheoretiker die Auffassung, dass kausale Relationen zwar Beziehungen zwischen Einzelereignissen sind, dass solche Beziehungen jedoch immer unter ein Gesetz fallen müssen, damit die Beziehung eine genuin kausale Beziehung ist. Kausale Beziehungen setzen also die Existenz von entsprechenden Gesetzen voraus. Und dass dies der Fall ist, soll aus dem Begriff der Kausalität selbst folgen. Kontrafaktische Theorien der Kausalität, wie sie z.B. von David Lewis vertreten werden, verstehen die kausale Relation als kontrafaktische Abhängigkeit der Wirkung von der Ursache. Demnach wäre die Wirkung nicht eingetreten, wenn die Ursache nicht aufgetreten wäre. Auch diese kontrafaktische Analyse der Kausalität soll sich aus dem Begriff der Kausalität ergeben. Sowohl die Regularitätstheorie als auch die kontrafaktische Analyse der Kausalität beanspruchen beide, begrifflich wahr zu sein. Was 
würde passieren, wenn eine dieser Analysen korrekt wäre? Könnte man immer noch daran festhalten, dass kausale Prozesse wahrnehmbar sind? Dagegen spricht die folgende Überlegung von Tooley und Sosa, die unter der Annahme der Regularitätstheorie formuliert wird: ,if (a causal) relation were an instance of some law, to observe that two events were causally related would be to observe that there was some relevant law, and it is not easy to see how a single obseravtion could serve to establish such a conclusion. “4 Man kann den Fall auch etwas verallgemeinern. Nehmen wir an, kausale Relationen würden begrifflich implizieren, dass ein Naturgesetz gilt oder dass eine bestimmte modale Abhängigkeit besteht. Dass ein Naturgesetz gilt, kann man nicht unmittelbar wahrnehmen. Einzelne Wahrnehmungen beziehen sich nur auf konkrete Einzeltatsachen. Sie haben niemals Gesetze zum Inhalt. Aber auch modale Abhängigkeiten kann man nicht wahrnehmen, denn man kann nur wahrnehmen, was aktual der Fall ist, nicht, was der Fall wäre, wenn etwas, das aktual der Fall ist, nicht geschehen wäre. Solche Tatsachen scheinen einfach zu weit von der Aktualität entfernt zu sein. Vielleicht kann man Annahmen darüber auf die Wahrnehmung stützen, aber es scheint nicht unmittelbar selbst Inhalt der Wahrnehmung zu sein. Also kann man Tatsachen nicht wahrnehmen, die durch den Begriff der Kausalität impliziert werden. Aber das läuft darauf hinaus, dass man Kausalität selbst nicht wahrnehmen kann. Diesen Einwand kann man mit Hilfe des folgenden Arguments der Geschlossenheit der Wahrnehmung unter begrifflicher Implikation rekonstruieren:

(1) Wenn eine Erfahrung repräsentiert, dass ein Ereignis a ein Ereignis b verursacht, dann muss sie auch alles repräsentieren, was begrifflich durch die Kausalrelation zwischen a und b impliziert wird.

(2) Kausalrelationen zwischen zwei beliebigen Ereignissen implizieren begrifflich schwergewichtige Tatsachen.

(3) Schwergewichtige Tatsachen lassen sich nicht wahrnehmen.

Also: Keine Erfahrung kann repräsentieren, dass ein Ereignis a ein Ereignis b verursacht.

Nehmen wir einmal an, dass (2) wahr ist, weil Kausalität begrifflich Gesetze oder kontrafaktische Abhängigkeiten impliziert (die ich als schwergewichtige Tatsachen bezeichne). Und nehmen wir ferner an, dass auch Prämisse (3) wahr ist, und Gesetze oder kontrafaktische Abhängigkeiten aus den genannten Gründen nicht wahrnehmbar sind. Sind

\footnotetext{
${ }^{4}$ Sosa/Tooley 1993, S. 13.
} 
wir dann nicht dazu gezwungen anzunehmen, dass kausale Prozesse prinzipiell nicht wahrnehmbar sind?

Nicht so schnell! Die Prämisse (1) drückt das Prinzip der Geschlossenheit der Wahrnehmung unter begrifflicher Implikation aus. Dieses Prinzip lässt sich bestreiten. Fred Dretske hat es zusammen mit dem Geschlossenheitsprinzip des Wissens angegriffen. Nun bin ich selbst der Auffassung, dass Wissen geschlossen unter begrifflicher Implikation ist. Wenn ich also weiß, dass eine bestimmte Proposition wahr ist, dann muss ich auch in einer Position sein, alles das, was logisch-begrifflich aus dieser Proposition folgt, zu wissen. Mit anderen Worten, würde ich eine logisch-begriffliche Implikation aus einer von mir gewussten Proposition ableiten, dann würde ich auch die abgeleitete Proposition wissen. Das ist sehr plausibel und ist vermutlich das Grundprinzip aller Argumentation. Aber bezüglich der Wahrnehmung bin ich ganz und gar einer Meinung mit Dretske. Er illustriert die Ungültigkeit der Geschlossenheit der Wahrnehmung unter begrifflicher Implikation mit dem folgenden Beispiel: Nehmen Sie an, Sie sehen einen Keks in einem Glas. Dass sich ein Keks in einem Glas befindet impliziert begrifflich, dass es materielle Gegenstände in der Welt gibt und dass der Idealismus falsch ist. Doch Sie können nicht sehen, dass es materielle Gegenstände in der Welt gibt oder dass der Idealismus falsch ist. Das sind unbeobachtbare Implikationen ihrer Beobachtung. Die verwendeten Begriffe sind viel zu abstrakt, als dass sich Inhalte Ihrer Wahrnehmung mit Hilfe dieser Begriffe charakterisieren ließen. Doch wenn sich mit Hilfe von unkontroversen Beispielen zeigen lässt, dass das Geschlossenheitsprinzip für die Wahrnehmung nicht gilt, dann darf man es in einem Argument gegen die Wahrnehmbarkeit kausaler Prozesse auch nicht verwenden. ${ }^{5}$

Das kann jedoch nicht das letzte Wort sein. Es mag sein, dass man eine Tatsache sehen kann, ohne alles zu sehen, was sie begrifflich impliziert. Aber es wäre eigenartig, wenn man behaupten wollte, dass man eine Tatsache auch dann sehen kann, wenn man nichts sieht, was sie beinhaltet. Es stellt sich also die Frage, was man positiv sieht, wenn man kausale Prozesse sieht, deren modale und gesetzesartige Implikationen man nicht sieht. Und die Antwort auf diese Frage kann nicht lauten, dass man die Kontiguität der Ereignisse sieht. Denn wir wollen ja gerade sagen, dass der phänomenale Eindruck der Kausalität sich von dem der Kontiguität unterscheidet. Hier muss man wohl sagen, dass der Sinneseindruck von kausalen Prozessen so etwas wie die Aktivität oder Dynamik der Ereignisse repräsentiert, die modale oder

\footnotetext{
${ }^{5}$ Ansätze zu einem solchen Argument finden sich in Siegel Ms.
} 
gesetzesartige Implikationen haben. Zweitens stellt sich die Frage, wie wir den Begriff der Kausalität durch Erfahrung erwerben können, wenn wichtige Elemente des Kausalbegriffs selbst nicht wahrnehmbar sind. Auf diese Frage kann ich keine endgültig überzeugende Antwort anbieten. Aber vielleicht hilft die folgende Analogie weiter. Ich hatte vorhin gesagt, dass man die Existenz materieller Gegenstände nicht unmittelbar wahrnehmen kann, weil es sich bei dem Begriff MATERIELLER GEGENSTAND um einen zu abstrakten Begriff handelt. Dennoch kann man diesen Begriff aus den wahrgenommenen Tatsachen abstrahieren. Vermutlich ist es mit schwergewichtigen Elementen des Kausalbegriffes ähnlich. Wir können sie als solche nicht wahrnehmen, aber aus dem, was wir wahrnehmen (nämlich der Aktivität der Ereignisse) abstrahieren.

Mit diesem tentativen Ausblick möchte ich schließen. Es hat sich gezeigt, dass sich Humes vermeintliche knock-down Argumente gegen die Wahrnehmbarkeit kausaler Prozesse durchaus entkräften lassen. Außerdem sprechen die experimentellen und phänomenologischen Befunde dafür, dass Kausalität tatsächlich durch unsere Erfahrung repräsentiert wird. Und da das Prinzip der Geschlossenheit der Wahrnehmung unter begrifflicher Implikation nicht plausibel ist, folgt aus der Tatsache, dass wir bestimmte schwergewichtige Implikationen kausaler Beziehungen nicht wahrnehmen können, nicht ohne weiteres, dass wir kausale Prozesse nicht wahrnehmen können. Am Ende ist die These der Wahrnehmbarkeit kausaler Prozesse doch nicht ganz so unplausibel, wie die Mehrheit der Philosophen gegen den Common Sense immer noch annimmt.

\section{Literatur}

Anscombe, G.E.M. 1971: „Causality and Determination“, in: E. Sosa / M. Tooley: Causation, Oxford UP 1993, S. 88-104,

Hume, David 1993: Eine Untersuchung über den menschlichen Verstand, Hamburg: Meiner. Ducasse, C.J. 1926: „On the Nature and Observability of Causal Relations“, in: E. Sosa / M. Tooley: Causation, Oxford UP, S. 126-136.

Menzies, Peter 1998: "How Justified are the Humean Doubts about Intrinsic Causal Links?", in: Communication \& Cognition 31, S. 339-364.

Siegel, Susanna Ms.: "The Visual Experience of Causation”, online.

Michote, Albert 1963: The Perception of Causality, London: Methuen.

Sosa, Ernest / Tooley, Michael 1993: Causation, Oxford UP. 
